\title{
Managing and Assessing Group Work from a Distance
}

\author{
Álvaro Figueira and Rui Pereira \\ CRACS \& FCUP \\ University of Porto \\ Porto, Portugal \\ $\{$ arf,resp $\} @$ dcc.fc.up.pt
}

\begin{abstract}
Group work is an essential activity during both graduate and undergraduate formation. Students develop a set of skills, and employ criticism which helps them to better handle future interpersonal situations. There is a vast theoretical literature and numerous case studies about group work, but we haven't yet seen much development concerning the assessment of individual group participants. It is not always easy to have the perception of each student contribution to the whole work. Nevertheless, more than frequently, the assessment of the group is transposed to each group participant, which in turn results in each student having the same final mark.

We propose and describe a tool to manage and assess individual group work taking into account the amount of work, interaction, quality, and the temporal evolution of each group participant. The module features the possibility to create two types of activities: collaborative or cooperative group work. We describe the conceptual design of our tool and present the two operating modes of the module, which is based on events, alerts and conditions. We then describe the methodology for the assessment in the two operating modes and how these two major approaches can be deployed through our module into pedagogical situations.
\end{abstract}

Keywords_-group work, individual assessment, online, tool.

\section{INTRODUCTION}

In many European countries the student / teacher ratio has been continuously increasing in past couple of years, mainly due to a more rational use of resources, as some would say; or measures to cut off public expenses, as another might say. Apart from the reasons, there is a clear need for the use of other pedagogical resources and pedagogical means in order to promote a more decentralized education. In this article we present and describe a tool to manage, promote and help to assess, learning activities developed in a group. Our view is that those activities should be increased because they surely are a possible, simple solution, to circumvent the ratio problem.

The interaction between students, while undertaking a joint project, provides them not only with an opportunity to have a more active role in their learning, but also a way to develop their social skills. In fact, the group work is a teaching strategy that makes students become more responsible for their learning and for the learning of their group fellows. This inherent responsibility, when used with care, is also a clear add-on to the development of the student's "soft-skills".

Although the concept of "collaborative learning" is quite attractive in the first instance, it appears that it presents certain difficulties for the teacher, especially at the time of evaluating the students. We recall that it is not always easy to have the perception of the contribution of each student to the whole group work. Accordingly, the adoption of this kind of pedagogical activity justifies the need for reliable instruments and techniques to provide a greater accuracy (and legitimacy) when assigning the student's final grading.

The most common difficulties associated with group work are [1]:

- A largely unequal contribution of the group participants

- An inability of the students to manage the different ideas and opinions while progressing

- A decentralization of the objective of the work due to the requirement for increased autonomy and control over the choice of information and its processing

- An individual assessment of each group participant

And, despite the obstacles referred are focused primarily on the student role in the development of group work, teachers themselves feel some difficulties, namely to evaluate, assess and grade each individual group participant. Together all these difficulties may actually hamper the accomplishment of activities involving collaborative approaches. Curiously, the most well-known learning management systems (Blackboard, Moodle and Desire2Learn) at this time of writing do not provide in their features portfolio a tool to distribute work among group participants and to individually assess them.

In this context we propose a new tool to help the teacher in the process of creating, maintaining and assessing online group work, with emphasis on the assessment of the individual participation.

In the following sections we describe the problems and perspectives related with group work (section two); in section three we present a survey regarding the understanding of the use of group work in our department. Based on these prior sections we describe the conceptual design of our tool, which is 
based on events, alerts and conditions (section four). In section five we describe the configuration aspects of the tool illustrating it with screenshots. Section six is devoted to the assessment in the two operating modes. Section seven relates our tool with some examples of real-world pedagogical situations, and we conclude the article (section eight) with a brief description of our tool, presenting its strengths and weaknesses, and directions for future work.

\section{GROUP WORK}

Nowadays, it is generally accepted that completing a work individually or in a group with other students typically influences the learning outcome, the learning of others and, eventually, the final grade of the student. Modern pedagogy [1], [11] takes into account the need to expose students to collaborative work. However, group work is still far from being consensual and reluctance to its adoption derives from many handicaps of the respective assessment procedure. In the next sections we discuss the merits of group work performed collaboratively; we list some of the most common problems associated with this learning strategy and the recent approaches to deal with it.

\section{A. A simple dichotomy: group work vs. individual work}

As described in Hoffman \& Rogelberg [2], one of the advantages of group work is that it provides students with an opportunity to improve their "social component", which is essential given that we are in the "social networks generation". Other more objective aspect of the group work is the fact that every student has the opportunity to acquire new learning, according to their knowledge / experiences and the knowledge / experiences of the colleagues, which otherwise would not be possible.

In 2007, Strijos and Fischer [3] presented a study about the challenges related to collaborative learning, its effects in the learning process and the benefits taken from it.

However, there are also disadvantages in conducting group work strategies. The main disadvantages can occur due to the large discrepancy with respect to the workload, or the engagement of each of the participants. In particular, it is related to the difficulty in assessing group work. The second drawback, taken from Kagan [4] is, according to the author, the common knowledge that determines the choice of this type of activities. More than often, not only the teacher but also the students prefer individual work situations to avoid large discrepancy in the assessments. To minimize these disadvantages some tools are used to analyze the development of the work, and record the interactions that are made [5]. We decided to include this functionality in our tool. Although the grading is not automatic, a list of interactions, and the purpose of these interactions are recorded and a synthesis is provided to the teacher.

\section{B. Two visions of group work: collaborative vs. cooperative}

The collaborative work is based on the vision that the work is carried out by applying systematic transformations from an initial state by all group members, up to a state commonly accepted by the group as "final". On the other hand, the cooperative work is based on perspective of a "pipeline" where the objectives can be divided into several tasks and from that conception that there can be a dynamic distribution of tasks among the group members.

\section{Assessment of group work}

Assessment is many times one of the most influential aspects when creating a new pedagogical activity. The way the work is evaluated and assessed largely depends on the purpose of it [6].

Taking into account the characteristics inherent in group work, it is essential to consider not just the final work but also the contribution of each element in the preparation of this work. We believe that there is a general consensus on respect to the need for differentiating the grades of participants in a group work. However, the same is not true when we discuss how this differentiation should be made. In general, it is considered that there are three aspects that should be taken into account in any collaborative activity: the distinction between individual and group accomplishments, the transformation that will occur throughout the duration of the collaboration and, the social, motivational and cognitive aspects [7]. Unfortunately, all of these characteristics are very hard to be objectively assessed in a short period of time.

However, there are many objective suggestions, for instance through auto and hetero evaluation [8]; through the creation of individual portfolios [9] and even through the use of basic level generic log-files, trace data, and "digests" to determine the interactivity degree in the course of the collaborative activity [10]. We took this later approach by automatically creating a statistical digest of the interactions, and presenting a list of comments describing the changes made by the group participants.

Knowing which strategy to adopt is still an open question, since every task has its own characteristics, namely on the modus operandi of group work, which makes it difficult to find a way that can be considered simultaneously advantageous to all the different cases.

Despite the relevance of each of the aspects mentioned, it is important that the assessment should be as objective as possible. The problem is that some of aforementioned parameters are inherently subjective.

Therefore, our view was to create a tool which could assist the teacher during the creation, management and evaluation of group work, with emphasis on the distinction of the individual contributions of group participants, but also considering the changes that occurred during the accomplishment of the work as a whole.

\section{Online group work}

The Moodle system is perhaps the most well-known and most-used worldwide, open source, learning management system. In Moodle there are different resources and activities that can be used during a course. In general, a resource does not require the intervention of the student to manipulate it. On the other hand, an "activity" does require the intervention of one or more students, and require the production, or 
participation in a given task. The Moodle standard package provides the following activities: Database, Chat, Forum, Glossary, Survey, Lesson, SCORM Package, Survey, Quiz, Assignment, Wiki and Workshop. There are activities which can be performed simultaneously by several students, whereas activities such as Test, or Survey, typically require individualized participation, although there may be some interaction with colleagues. It is curious to note that while it is possible to monitor the students' work in most Moodle activities, in none of them (except the Wiki) the teacher is able to monitor the participation of each student individually. If we consider other LMS which feature collaboration tools, we get essentially the same problems [11]. Hence, we may state clearly that in the most well-known LMS there is missing one activity where students may work collaboratively and be assessed individually.

\section{SURVEY ABOUT GROUP WORK AND ITS ASSESSMENT}

We conducted a small survey in our department intending to know which colleagues use group work methodology and how it relates to using automatic systems to compute the grade of each group participant.

The survey was distributed to all Department members and to all colleagues from other Departments that give lectures in our Department. From a universe of 53 teachers we received 44 filled questionnaires. We then discarded three of them due to some inconsistency in the values (the totals did not match by one or two units). Therefore, the following tables are built from a respondent universe of 41 teachers.

\section{TABLE I. USE OF GROUP WORK.}

\begin{tabular}{|l|c|c|}
\hline & Yes & No \\
\hline Use group work & 33 & 8 \\
\hline Use online group work & 18 & 15 \\
\hline Automatic assessment & 4 & 14 \\
\hline
\end{tabular}

Table $\mathrm{I}$ is a clear indicator that group work is a pedagogical strategy currently in use, as it is used in a great diversity of courses (33). Interestingly, only half of those use group work supported by online environments and, only in 4 courses it is used the automatic assessment provided by the online tool that supports group work. This situation reflects the lack of available technology to support and assess group work.

\section{TABLE II. NUMBER OF STUDENTS IN EACH GROUP.}

\begin{tabular}{|c|c|c|}
\hline Students in a group & № & $\%$ \\
\hline 2 & 9 & 27 \\
\hline 3 & 14 & 42 \\
\hline 4 & 3 & 9 \\
\hline 5 & 2 & 6 \\
\hline more than 5 & 1 & 3 \\
\hline diversity of size & 4 & 12 \\
\hline
\end{tabular}

In Table II we can see that groups of three participants are the majority, being followed by groups of 2 participants. Given our lecturing experience we tend to guess that this reduced number of participants is due to the inherent difficulty in assessing properly the amount of work and involvement of each participant in group work.
From Table III we conclude that even though each submitted group work is graded as a whole, teachers tend to try to grade differently the group participants using some factor(s), or characteristic(s), which are external to the online tool used.

TABLE III. METHODOLOGY TO GRADE INDIVIDUALLY GROUP MEMBERS.

\begin{tabular}{|l|c|c|}
\hline How to grade individ group work? & № & \% \\
\hline Same grade & 5 & 15 \\
\hline Same grade + adjustments & 11 & 33 \\
\hline Depends on oral presentation & 13 & 39 \\
\hline Other combination & 4 & 12 \\
\hline
\end{tabular}

This situation reflects the need to provide the evaluator with more information about how group work has been carried out. We stress that group work cannot be an atomic task performed by several students without any kind of feedback to the teacher and to the evaluator.

\section{CONCEPTUAL DESCRIPTION OF THE TOOL}

Our concern was therefore to design a computer application that assists teachers in creating, managing and evaluating group activities. Conceptually the tool should provide the infrastructure needed for any normal pedagogical activity that can be performed in a group through a web-based system. Thus, disregarding the specific interface design and programmatic principles, we assemble the following guidelines for such a system:

- Be able to distinguish between a task and different subtasks, i.e. between a final product in which there is no specification of intermediate steps, and also the opposite situation. In this sense we created two different modes of operation: the "sequential activity" and the "simultaneous activity".

- Be able to set deadlines for submissions sub-tasks (if they exist) and of the final product; also be able to establish deadlines for assessment phases; create a default procedure in case of violating these deadlines.

- $\quad$ Be able to help students set intermediate milestones associated with the tasks.

- Allow group participants to discuss, comment and evaluate their processes along the work; be able to gather all these comments and present them to the teacher if needed.

- Allow the retrieval of data automatically collected, together with usage statistics, to help the teacher in understanding the contributions of each group participant regarding the final product submitted.

Based on these guidelines we developed a tool that can be used in Moodle as a new module. The tool allows the creation of batch jobs automatically, managing the group's actions, and helps to conduct the evaluation of each work group. Specifically, the system's interface allows the teacher to follow the interventions of each individual student, as well as the group as a whole. Accordingly, using this information the teacher can also make comments and give feedback to students during their work. 
Regarding the evaluation, the tool allows the teacher to give an individual grade to each group member, based on the amount of work computed and on the automatic report created, as well as on the contributions made to help other group participants. Also, since this is a group activity, the teacher also has the opportunity to submit a grade to assess the performance of the group itself.

By using this tool the teacher can observe the performance of each group participant along his/her proposed resolution of the problem and, along with other information provided by the system. Therefore, when having to grade each student, the teacher would have more insight in assigning a score to each group participant.

\section{A. Sequential activity}

The first type of the activity we propose is called the "sequential", or "linked", activity, whose main characteristic is that it allows a job to be divided into several tasks distributed by the various group participants, each participant responsible for a particular task.

This activity is conceptually described in the diagrams shown below. As illustrated in Figure 1, there is a deadline represented by a dashed line (1), for the whole work. That is, all the tasks should be completed within the period established by the teacher during the activity creation. When a single task is completed, the system will present automatically the next task in the list (provided it is not the last one). After the completion of the last assigned task, some (optional) improvements are allowed, and the system ends the activity.

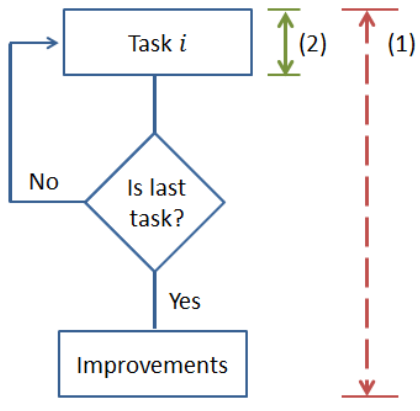

Fig. 1. Generic diagram of the sequential activity.

In Figure 2 we describe what happens within each "task". One of the deadlines, represented in Figure 1, labeled as (2), and also represented on the left hand side of Figure 2, as an arrow, is the duration within which student $j$ must complete task $i$ assigned to him. The teacher sets the deadline for this task and, if the student does not finish it in time, the task will be assigned to student $j+1$. The second deadline concerns the evaluation period. The group members must evaluate the submitted task within a certain interval of time resulting in the group approval (or not) of this task. The approval may take place within one of four criteria set by the teacher: "unanimity", "majority", "threshold" and "teacher assessment". Unanimity is a mode in which the work is approved if all group members approve it. If the criterion is set to approval by a majority, then a task is approved if the majority of the group approves it (in case of a tie, the teacher should intervene to break it). The "threshold" is a mode in which each of the group participants assign a grade to the submitted task, and this will be approved if the average grade is greater than or equal to the value set by the teacher. Finally, if established by the approval of the teacher, a task will be approved regarding only the teacher's opinion.

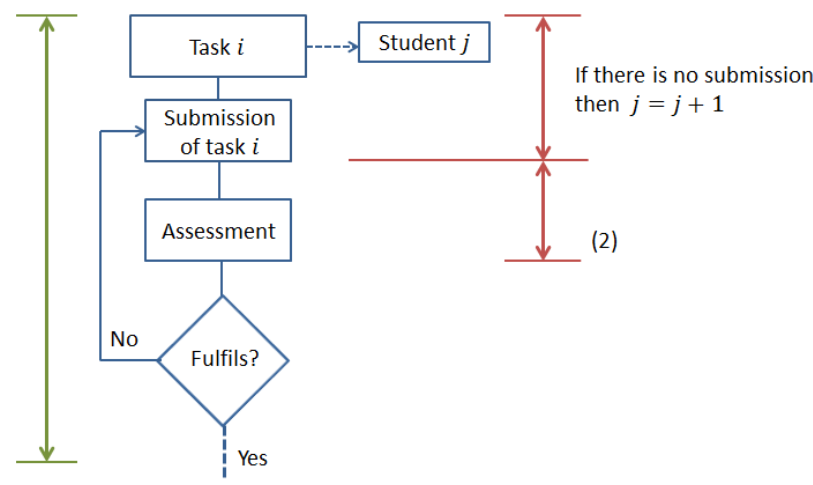

Fig. 2. Generic diagram of the sequential activity with focus on the task.

\section{B. Simultaneous activity}

The second type of activity assumes that all group members can freely interact with each other and simultaneously changing the product of their group work. While the interaction of group members with the resulting product is controlled by the system, the interaction between group participants is not. However, all changes that are made in terms of the final product are registered in system. That is, our tool creates in Moodle a "store" for each group, which then houses their set of data / files. During the time allocated to the group work this set is incremented, changed, or simply manipulated until it reaches a state considered satisfactory by all group participants. Then, from the general agreement of the group it may then be submitted as the group "final product". This process is illustrated in Figure 3.

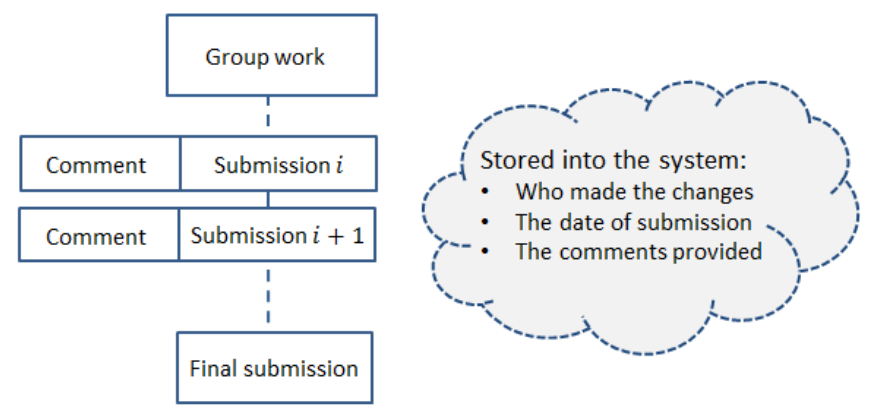

Fig. 3. Generic diagram of simultaneous activity.

\section{DESCRIPTION OF THE TOOL, ITS CONFIGURATION AND USE}

Our built system features the possibility to create collaborative or cooperative work. It is up to the teacher to decide which suits best, at any pedagogical case, and configure it from the initial administration panel. 
According to Moodle standards, the creation of an activity begins by setting its title and description. Then, the teacher selects one of the two possibilities: "sequential" or "simultaneous" activity type, and establishes the initial and final deadlines for the completion of the activity, as in Figure 4.

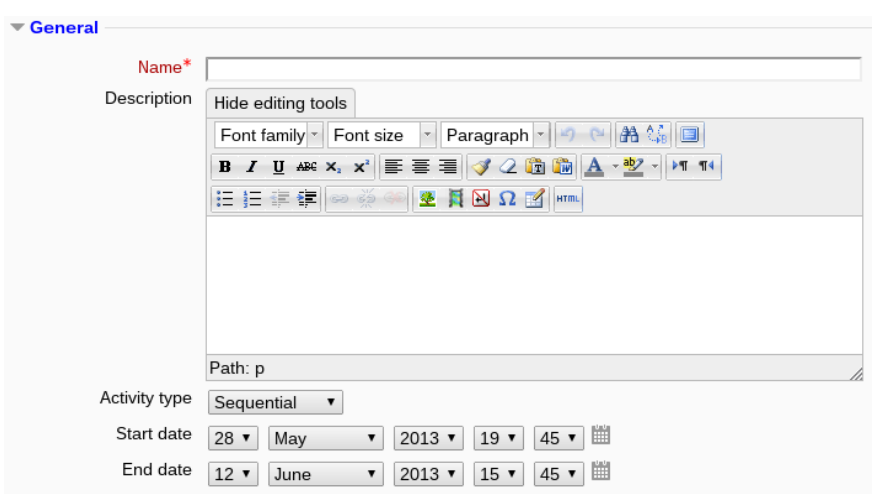

Fig. 4. Creation of a collaborative group activity - selecting sequential mode.

\section{A. Sequential activity type}

This format has as main characteristic the fact that the tasks are distributed among all group members, ie, each student will work in a different part of the whole group work. All student get access to the general description of the work, but the student responsible for the first task will be the only to have access to the description of that task. As suggested in Figure 5 and Figure 6, during activity configuration, the teacher sets the number of tasks that are appropriate, taking into account the number of group elements and the characteristics of the whole job to be undertaken by the group.

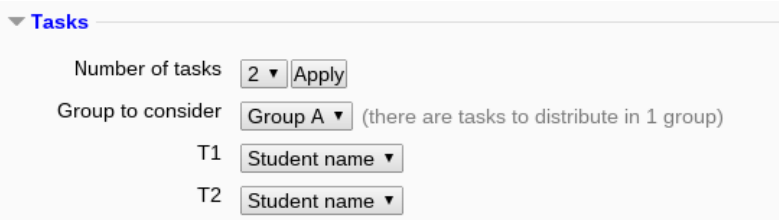

Fig. 5. Distribution of tasks among group participants.

The teacher must also assign a deadline for the completion of tasks, which may be the same for all of them (first option shown in Figure 6), or set specific deadlines for each task (second option illustrated in Figure 6). If the teacher chooses the second option, the system will present a list of tasks which will then have to be filled with the respective duration (in hours). This last option may be used in situation where the expected duration of a task in not proportional to the effort needed to complete it. For example, finding a good set of references usually takes much more time than compiling them in a web page, to give just an example.

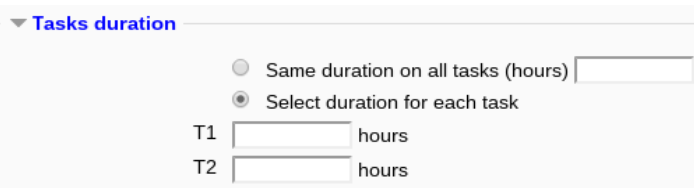

Fig. 6. Setting task duration.
When any task is submitted all other group participants will have access to the work of the first participant, and must approve or disapprove it. Note that the teacher can set a deadline for that assessment to avoid spending too much time with the evaluation of submissions. The grading takes place according to four possibilities (Figure 7):

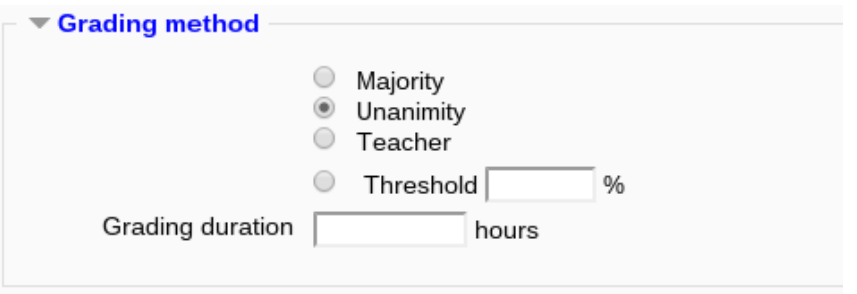

Fig. 7. The grading criteria.

A negative opinion on a colleague's work should be associated with a comment of how to improve it. This comment is then made visible to all group participants. If the submission is approved and submitted within the due time, the next task will be assigned to the next student. This process is repeated until there are no more tasks to assign. If the work of a student is not approved, even after its redesign, the teacher should intervene and help the group to move forward with this work.

If student $j$ does not submit the job within the due time, it will be assigned to student $j+1$. The group participant that has not fulfilled his task will be assigned to the last task (eventually having a penalty established by the teacher) and there will be a "rotation" of tasks.

Associated with the submissions engine there is an automatic type of forum which students and teacher use to register comments to the work done by each group (separately).

If one or more tasks are unfulfilled, they will be available to all members of the group who have completed their tasks, to allow the group to conclude the proposed work. In the end, there is also a period of time to make specific improvements in each of the tasks submitted.

\section{B. Simultaneous activity type}

The second format is what we call "simultaneous work", meaning a joint activity. In this mode group members are allowed to build together the final work. Basically, the principle behind this mode is that the interaction between students is a "black box", and the only visible actions of the group are changes made to a set of files that in the end will be the submitted product of this group. In Figure 4 we presented the configuration screen of this activity. This mode is chosen basically by selecting a different activity type.

Once assigned a task to a group, the participants can submit new files and/or change files already submitted. The system saves all submissions made to the respective versions of each file, the author of each submission, the date of the submission, and the comments given by the student who made the changes. This is reflected on the table presented in Figure 8 (visible both to the teacher and to each participant of this group). 
For each file submitted, it is possible to access all of its versions through the respective link in the text (the dates), which has indicated the date and time of that submission and, who made that submission. It is possible to access the latest version of each file by clicking on the leftmost link (date). The upload button (with an arrow pointing upwards) is used to perform an upload of a new version of the respective file. There is also the possibility to view all comments made by students on each file by pressing the "i" button.

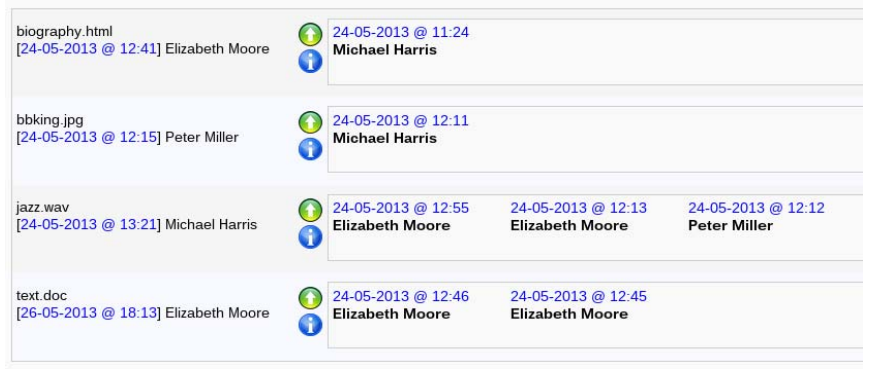

Fig. 8. Table illustrating file versioning during a collaborative activity.

Basically, the simultaneous collaborative activity unfolds like this: a task is presented to each group of students, eventually requiring them to confront their ideas, to reach a consensus with the aim of ultimately submitting a set of file which express and describe coherently the group ideas. To reach this end, the group participants are expected to save in the group online repository files that are continuously being manipulated by them until they reach a ready state to be submitted. Hence, there is a virtual place where students submit temporary files to which the rest of the group has access. During the progress of the work, the changes to these files are logged by our module, which is therefore used as a versioning system.

In Figure 9 we show a possible interface display for student "Elizabeth Moore". As the figure suggests, she may submit a new file to the group repository, which should be accompanied by a commentary (in field "description").

On the right hand side of Figure 9 the interface presents the "Allow submission box". As the group works collaboratively and simultaneously, it is important to know at which stage the current work is able to be submitted. Clearly, this is a decision to be taken by all group participants. Accordingly, the system provides a democratic mechanism in which every participant must grant permission for the submission of their group work. Only after permission has been granted by all group members, the "Submit work" button is displayed. The moment all students have grant authorization for final submission, all names listed are followed by "OK", and the "Submit work" button will be available. In Figure 9 we can see that students Michael Harris and Peter Miller have not yet authorized the submission of the work.

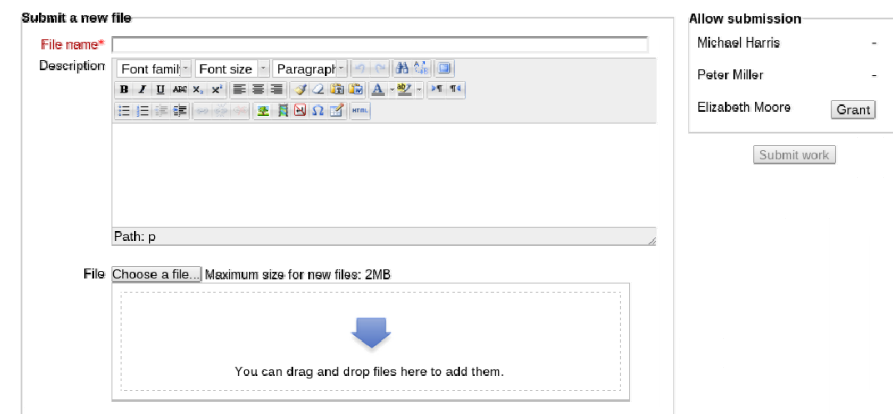

Fig. 9. Submission of a new file and the granting permission for final job submission

\section{STUDENTS ASSESSMENT USING THE ONLINE TOOL}

Given that there are two distinct modes for the activity, sequential and simultaneously, we define some basic rules for the evaluation of each group participant, in each mode.

\section{A. Assessment of the sequential activity}

In this mode, as we saw earlier, each student performs one or more tasks individually, which will then be evaluated by all members of the group. After the work is validated by all students, the system launches a new task to be performed by another group participant, following a previously established ordering of the pairs (student-task), set by the teacher. The final grade is assigned using the same weight to each task, so that all students have the same conditions (recall that the need to set duration for a task is related with the task's difficulty or with the amount of work for that task. For that reason it is important to have this parameter as fair as possible). On the other hand, the final score for each student should take into account the following parameters:

- Quality and appropriateness of the work submitted

- Capacity to meet the deadlines

- Capacity of evaluation of the tasks carried out by colleagues and respective comments

- Final score of the work

These parameters intervene in the final assessment using the Moodle's well known 20\% variable offset for grading. I.e., the teacher may adjust the final score up to $20 \%$ from the computed grade.

We recall that from the initial configuration of the sequential activity it is possible to choose an evaluation strategy based on the assessment mode: it can be either based on unanimity of opinions (yes/no) about a task; on a majority of these opinions (yes/no); on a teacher's assessment (in percentage); or, using a threshold percentage value. In Figure 10 we present a diagram illustrating how these four possibilities lead to an individual grade for each group member. 


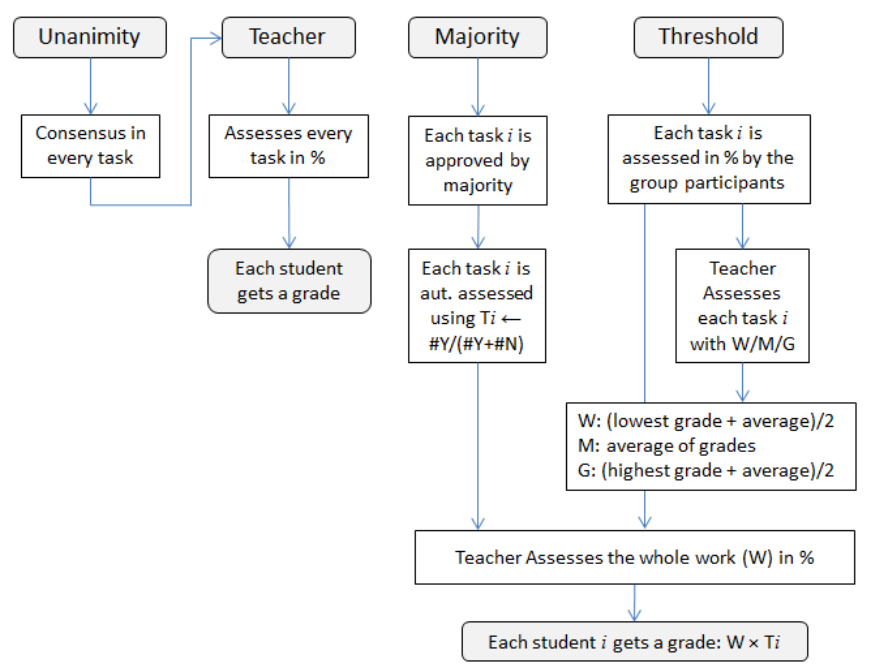

Fig. 10. Grading each group participant in the sequential mode.

As can be seen on Figure 10, the unanimity mode extends the teacher mode with a consensus of all group participants before submission of each task. Using the threshold and majority mode, the final grade for each group participant is influenced by the grade given by the other group participants and the grade given by the teacher. In mode "majority" the teacher only grades the whole work, whereas in mode "threshold" he/she has another possibility: to assess each task using a three-level scale - weak, medium or good. Each of these three levels act as an aggregator to the grades given by the group participants, which finally will be combined with the final grade given by the teacher to the whole work.

In Figure 11 we present the evaluation screen for the teacher using the threshold mode.

\begin{tabular}{|c|c|c|c|c|}
\hline & & Evaluatlon & & \\
\hline & Whisle Wrork. & 36 & Fval ıate & \\
\hline Taaka & 3laie of fask & Itote of evaluallen & Craluallen & \\
\hline Tasl: 1 & complasd & Arallable tor evaluaton & $w \circ G$ & Fvaluatf \\
\hline Iastic 2 & Somplafsd & Frallable tor evaluaton & $O \mathrm{WO}, \mathrm{g}$ & Evaluate \\
\hline Tazk: : & Somplatad & Ayallabla for axaluation & W० $M O G$ & Evaluetc \\
\hline Tazk A & Complatad & Evaluatbon corrp $\theta, \theta$ & $0 \mathrm{w0} 1 \mathrm{MO}$ & Evaluate \\
\hline
\end{tabular}

Fig. 11. Sequential activity, threshold mode, teacher evaluation.

\section{B. Assessment of the simultaneously activity}

In this type of activity group participants perform tasks that they consider appropriate to fulfill the job proposed to the group, rather than having a set of pre-defined and assigned tasks. The responsibility to distribute work and continuously assess the state of its quality relies on the group participants exclusively. Accordingly, it is not important the intermediate stages of the work. There can be three stages, ten or a hundred - it does not matter. What matters is the quality of the final product, and the involvement of each group participant during the working period. Therefore, the evaluation of this activity must be assessed globally by the quality of the submitted product, and individually using indicators of the contribution of each group participants to this final product.

In Figure 12 we present the final evaluation screen for the simultaneous activity, for one single group. The teacher is able to see for each group participant the number of created files, changed files, the daily frequency of changes and comments made by that participant. Apart from the frequency, all values are links to the respective list where the file, or comment, can be accessed. Using this information the teacher should provide a grade for each student.

\begin{tabular}{|c|c|c|c|c|c|}
\hline \multicolumn{6}{|c|}{ Evaluation } \\
\hline Student & Created filer & Changed files & Frequency & Comments made & Evaluation \\
\hline Ky: Fhllllps & 4 & 3 & 0,29 & 6 & $\square \%$ \\
\hline Jaan Taylor & 3 & 3 & 0,26 & 3 & $\square \%$ \\
\hline Emilly Worrlg & 3 & 2 & 0,21 & 3 & $\square \%$ \\
\hline Wegan Parker & 5 & 1 & 0,26 & 3 & \rceil $\%$ \\
\hline & & & & & \begin{tabular}{|l|} 
Evaluate \\
\end{tabular} \\
\hline
\end{tabular}

Fig. 12. Simultaneous activity evaluation screen.

Thus, the final grade of each student takes into account his/her involvement during the group work as well as comments given. We deliberately did not use more parameters because it is generally difficult for someone to respect a rationale based on more than four variables.

\section{PARALLEL TO REAL-World PEDAGOGICAL SITUATIONS}

The use of group work if often confused with collaborative, or even cooperative, work. Interestingly, as a pedagogical tool, group work has largely been promoted in most e-learning environments. Forums, wikis and workshops are usually referred as tools to be used collaboratively. However, none of these examples is actually a tool to manage group work because they do not provide a means to assess each participant individually. On the other hand, the primary objective of every major e-learning platform, in the last decade, was to have some kind of tool to promote and foster collaborative/cooperative work.

The interactions between group participants are often seen as fundamental during group work [12], not only for the development of the product, but also as anchors for the teacher to understand problems and contributions from the students. Our tool also helps students to keep a record on their interaction regarding changes to the groups' final product. In the literature auto and hetero-evaluation is also regarded as important [13] in order, not only to increase interaction between participants, but also to improve the final group grade. 
As a consequence, we included in our tool several modes which force an assessment of each group participant. However, as seen on [10] it is important to have a tracking system, or "log files" to understand the "interactivity intensity", and that is precisely what our tool offers to the teacher.

In a short synthesis, the tool we propose in this paper has a large set of applications to real-world pedagogical situations due to the two main activities and to the set of assessment modes. For example, in Horton [14]: guided-research activities are well suited for our approach of the sequential perspective with evaluation being based on consensus; web-quests are another example of sequential activities but probably, in this case, the threshold evaluation mode would be more convenient; the "treasure hunt" or the "story-telling" activities are naturally designed to be supported by a collaborative tool, thus, in this case the simultaneously activity should be used. Other examples can be presented but they inherently fall into a sequential or simultaneously activity.

\section{CONCLUSION}

Sometimes group assignments are merely a set of individual tasks, which later on are gathered and assessed as a single activity, where the participation and involvement of each group participant is seen as part of a "black box". More than frequently, the grading of each participant for his/her developed work is not meritocratic, but just the same as the mark given to the whole group work, which makes it the same to all participants, disregarding each individual involvement.

In this paper we propose and describe a web-based system that allows the creation, administration and assessment of group activities with emphasis on the individual assessment of each participant.

Our system can help overcome the difficulties to evaluate the work and involvement of each group member by allowing the teacher to distribute group tasks, to analyze the outcomes of each task and to verify the participation of each student. We stress the fact that our tool has two types of activities is a distinct advantage for the teacher, since he/her can choose the type of activity according to the pedagogical objective to be reached within the course taught. Moreover, the tool allows the teacher to follow the progress of the group work, and give an opinion to unlock, if necessary, deadlock situations.

While preliminary results from real use indicate that the tool is used with efficacy, promoting group work and increasing the engagement of participants, a more detailed analysis is still needed.

Future work with this tool will involve: i) the analysis of a preliminary usability test conducted during the last month; and, ii) the discussion of the assessment results of the system in a real learning scenario, in order to prove the functionality of the tool as well as the expected benefits that derive from it.

\section{ACKNOWLEDGMENT}

Project "NORTE-07-0124-FEDER-000059" is financed by the North Portugal Regional Operational Programme (ON.2 - O Novo Norte), under the National Strategic Reference Framework (NSRF), through the European Regional Development Fund (ERDF), and by national funds, through the Portuguese funding agency, Fundação para a Ciência e a Tecnologia (FCT).

\section{REFERENCES}

[1] K. Swan, J. Shen, and S.R. Hiltz, "Assessment and Collaboration in Online Learning" in J. Asynchronous Learning Networks, vol. 10, no. 1, pp. 45-61, 2006.

[2] J. Hoffman, and S. Rogelberg, "All Together Now? College Students' Preferred Project Group Grading Procedures" in Group Dynamics: Theory, Research, and Pratice, vol. 5, no. 1, pp. 33-40, 2001.

[3] J. Strijos, and F. Fischer, "Methodological Challenges for Collaborative Learning Research" in Learning and Instruction. Vol. 17, no. 4, pp. 389394, 2007.

[4] S. Kagan, "Group Grades Miss the Mark," in Educational Leadership, vol. 52, no. 8, pp. 68-71, 1995.

[5] Y.J. Chyng, C. Steinfeld, and B. Pfaff, "Supporting Awareness among Virtual Teams in a Web-Based Collaborative System: The TeamSCOPE System," in ACM SIGGROUP Bulletin, vol. 21, no. 3, pp. 28-34, 2000.

[6] D. Boud, R. Cohen, and A.J. Sampson, "Peer Learning and Assessment". In Assessment and Evaluation in Higher Education, vol. 24, no. 4, pp. 413-426, 1999.

[7] J.W. Strijos, "Assessment of (Computer-Supported) Collaborative Learning" in IEEE Transactions on Learning Technologies, Vol. 4, no. 1, pp. 59-70, 2011.

[8] R. M. Gilies, "Cooperative Learning: Integrating Theory and Practice", Sage, 2007.

[9] E. Lee, C. Chan, and J. Aalst, "Students Assessing Their Own Collaborative Knowledge Building," in International Journal of Computer-Supported Collaborative Learning, vol. 1, no. 1, pp. 57-87, 2006.

[10] T. Schummer, J. Strijos, and T. Berkel, "Measuring Group Interaction during CSCL" in Proc. Conf. Computer Supported Collaborative Learning: Learning (CSCL ' 05), pp. 567-576, 2005.

[11] B. Burnett, and A. Roberts, "Online Collaborative Assessment: Unpacking Process and Product," in Assessing Online Learning, P. Comeaux, ed., pp. 55-71, Jossey-Bass, 2005.

[12] D.W. Johnson, "Student-Student Interaction: The Neglected Variable in Education," Educational Researcher, vol. 10, no. 1, pp. 5-10, 1981.

[13] J.A. Ross and C. Rolheiser, "Student Assessment Practices in CoOperative Learning," Co-Operative Learning: The Social and Intellectual Outcomes of Learning in Groups, R.M. Gillies and A.F. Ashman, eds., pp. 119-135, Routledge, 2003.

[14] W. Horton, E-Learning by Design. 2nd Edition, San Francisco, CA, Pfeiffer, inprint of Wiley, 2012. 